\title{
Study on bisphosphonate-related osteonecrosis of the jaw (BRONJ): case report and literature review
}

\author{
Yeo-Gab Kim¹, Baek-Soo Lee', Yong-Dae Kwon', Joon-Ho Suh', Sang-Mi Jeen² \\ 'Department of Oral and Maxillofacial Surgery, School of Dentistry, ${ }^{2}$ School of Dentistry, Kyung Hee University, Seoul, Korea
}

\begin{abstract}
J Korean Assoc Oral Maxillofac Surg 2010;36:291-302)
Bisphosphonate-related osteonecrosis of the jaw (BRONJ) is a side effect of Bisphosphonates (BPs) use. These days, oral bisphosphonates are normally prescribed to treat osteoporosis. Intravenous BPs are used extensively to treat osteolytic bone lesions related to multiplemyeloma and bone metastasis of solid cancers, breast cancer or prostate cancer. As the prescription of BPs is universalized and the number of people treated with BPs is increasing, an accurate understanding and proper management of BRONJ are required. The aim of this study was to improve the clinicians' understanding of BRONJ by reviewing the literature. To achieve this, this paper introduces case reports as well as the current concept of BRONJ based on the 2009 updates by American Association of Oral and Maxillofacial (AAOMS) including the definition, epidemiology, etiology, diagnosis, treatment and prevention of BRONJ.
\end{abstract}

Key words: Bisphosphonate, Osteonecrosis, Osteoporosis, Jaw diseases

[paper submitted 2010. 5. 6 / revised 2010. 7. 15 / accepted 2010. 7. 27]

\section{I . Introduction}

Bisphosphonate-related osteonecrosis of the jaw (BRONJ) has been known as a side effect of Bisphosphonates (BPs) since Marx ${ }^{1}$ in 2003. These days, Oral bisphosphonates like alendronate (Fosamax, Merck, westpoint, VA) are usually prescribed to treat osteoporosis and intravenous BPs are extensively used to treat osteolytic bone lesions related to multiple myeloma and bone metastasis of solid cancer, breast cancer or prostate cancer ${ }^{2}$. As the prescription of BPs is universalized and the number of people treated by BPs are increasing in the long term, the accurate understanding and proper management on BRONJ are required.

Although American Association of Oral and Maxillofacial Surgeons (AAOMS) has reported the severity of this disease by suggesting guidelines on BRONJ since $2006^{3}$, it is likely that dentists in Korea have little understanding of this'. Park et $a l .{ }^{5}$ surveyed the awareness of BRONJ targeting 226 Korean dentists. The results are as follows. 1) $45.1 \%$ of Korean dentists answered that they experienced healing retardation by

\section{김 여 갑}

130-701 서울특별시 동대문구 회기동 1

경희대학교 치의학전문대학원 부속병원 구강악안면외과

Yeo-Gab Kim

1 Hoegidong, Dongdaemungu, Seoul, 130-701, Korea

Department of Oral and Maxillofacial Surgery, Kyung-Hee University

Tel: +82-2-958-9440

E-mail:kyukab@khu.ac.kr exposed bone after extraction. Even though the lesions are evenly distributed on mandible and maxilla, that is, it' s totally different from the usual osteomyelitis, only $15.1 \%$ asked their patients whether they had been taking BPs. 2) The Korean dentists who have simple perception on BRONJ are $56.5 \%$. On the other hand, only $28.9 \%$ are aware of the possibility of BRONJ after bloody dental treatment is performed to the patients who have taken BPs. 3) 57.2\% know the severity of BRONJ. However, only $19.3 \%$ are aware of the suggestion of AAOMS on BRONJ. 4) There' s a tendency that the shorter they have clinical experience, the more they know about the BRONJ and the suggestion of AAOMS. But there' s no difference according as the amount of clinical experience in the awareness of the possibility of BRONJ after bloody dental treatment.

The goal of this paper is to improve the clinicians' understanding on BRONJ by organizing published researches. To achieve this, this paper introduces the case reports as well as the current concept of BRONJ based on 2009 updates by AAOMS including definition, epidemiology, etiology, diagnosis, treatment and prevention of BRONJ.

\section{II . Case reports}

On June 11th, 2009, a 74 year old female patient was transferred from a local clinic because the extraction wound failed to heal after the extraction of the maxillary right first premolar, maxillary left second premolar and second molar. 
This patient visited the local clinic due to pain during mastication in these areas. Severe alveolar bone loss was observed through clinical and radiographic examination. Consequently the teeth were extracted throughout April 13th and 14th. However the healing of the extraction wound was delayed. Despite performing curettage of the extraction wound on May 11 th and postoperative conditioning approximately 20 times, the symptoms did not improve and was referred to our department.

Medically, the patient was being treated for hypertension and diabetes, which were being efficiently controlled. The patient also had been administrating actonel (risedronate) for about 20 years, due to osteoporosis.

Delayed healing of the extraction wound and pus discharge, along with exposure of alveolar bone at the maxillary right first premolar and maxillary left second premolar and second molar area was observed during clinical examination of the initial evaluation.(Fig. 1)
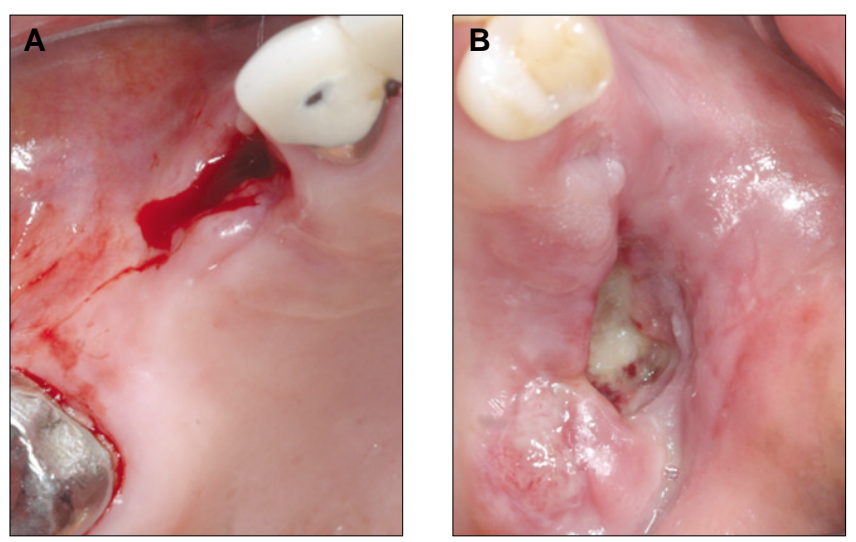

Fig. 1. A. Delayed healing of the extraction wounds with exposure of alveolar bone and pus discharge of the maxillary right first premolar. B. Maxillary left second premolar and second molar.

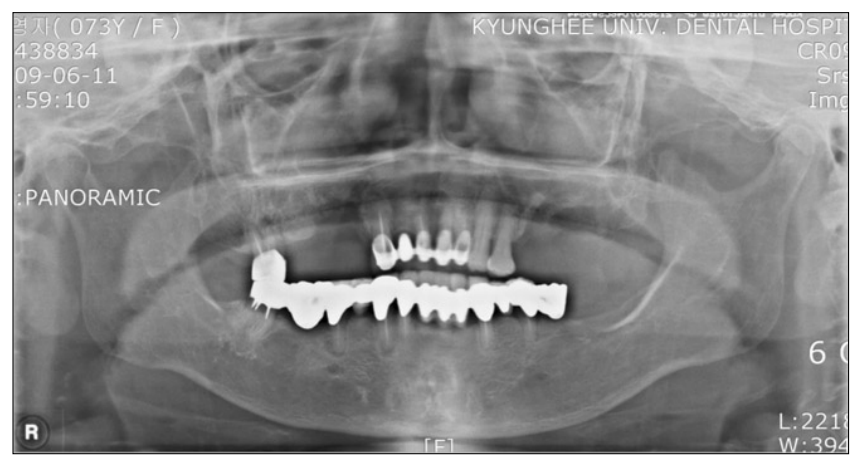

Fig. 2. Radiolucent status with ill-defined border of the lesion area is observed in the panoramic view of the initial examination.
Diffuse osteolytic appearance is found in the maxillary right first premolar, maxillary left second premolar and maxillary left first molar area on the panoramic view.(Fig. 2) Also radiopaque appearance was found on both maxillary sinuses on the water's view.(Fig. 3)

On the dental computed tomography (CT) (Fig. 4), osteolytic appearances with ill-defined borders were found in the maxillary left and right extraction wound areas, sequestrum formation was shown in the maxillary left area, and cortical bone destruction of the sinus floor was observed. The maxillary right region showed reactive osteosclerotic state and osteophytosis on the sinus floor, and radiopaque appearance inside the sinuses.

The display of osteomyelitis accompanied by bilateral sinusitis in the clinical and radiographic examinations, and the history of administrating bisphosphonate (actonel) due to osteoporosis for 20 years, together lead to the provisional diagnosis of BRONJ.

The treatment plan was determined as following.

1. In order to receive assistance considering the confirmed diagnosis of BRONJ, the current state of osteoporosis, and the administration of therapeutic agent for osteoporosis, consulting with the department of endocrine medicine which provides a co-operative treatment system.

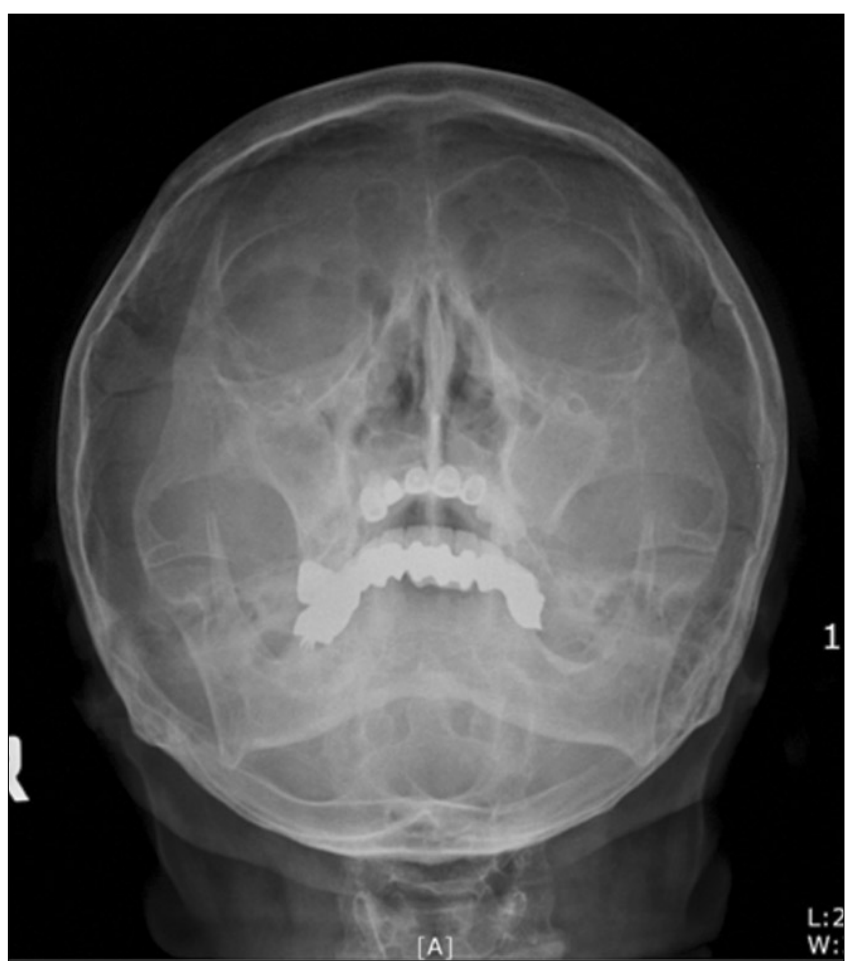

Fig. 3. Bilateral maxillary sinus area haziness is shown in the water's view of the initial examination. 

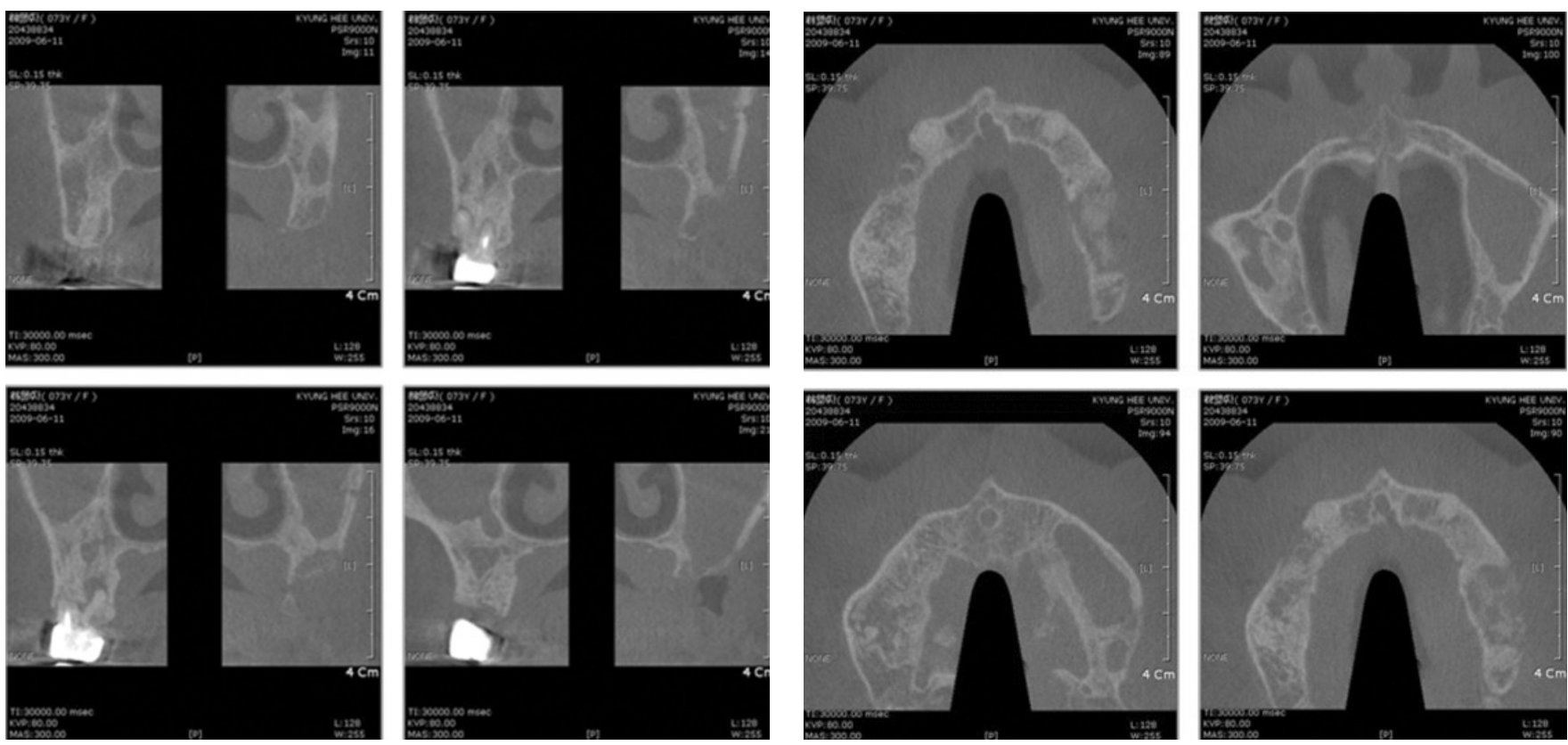

Fig. 4. Bone destruction status is observed in the maxillary left and right lesion area, sequestrum formation can be identified in the maxillary right lesion area, and reactive osteophytic status is shown in the sinus floor area in the lesion area of the maxillary left side in the dental computed tomography (CT) image of the initial examination.

2. Antibiotic treatment and mouthwash prescription for oral hygiene.

3. While performing conservative treatment, evaluate of the risk of operation and perform sequestrectomy.

The result of the endocrine examination showed low risk of BRONJ according to s-CTX level $(0.192 \mathrm{mg} / \mathrm{mL})$ (Roche Diagnostics GmBH, Penzberg, Germany), but very low level of 0.5 in osteocalcin (bone formation marker) was observed and the duration of actonel (risedronate) (Procter \& Gamble, Cincinnati, USA) administration was long. Therefore the overall risk of BRONJ occurrence was determined to be moderate to high.

Before performing the treatment, one must explain the postoperative problems of BRONJ to the patient. In other words, the patient must be told that although drug therapy and surgical treatment necessary, such as sequestrectomy, will be performed according to the position statement considering BRONJ presented by the institute, the treatment for BRONJ including drug administration characteristically takes time. The treatment of intraoral therapy and drug therapy was performed every week from the initial visit to September 18th, 2009. As new bone formation and definite sequestrum formation at the bone destruction area was observed in the radiograph taken after 3 months since the first visit (Figs. 5, 6), sequestrectomy was to be performed. The patient was hospitalized on October 5th, 2009 and received sequestrectomy and biopsy, then was

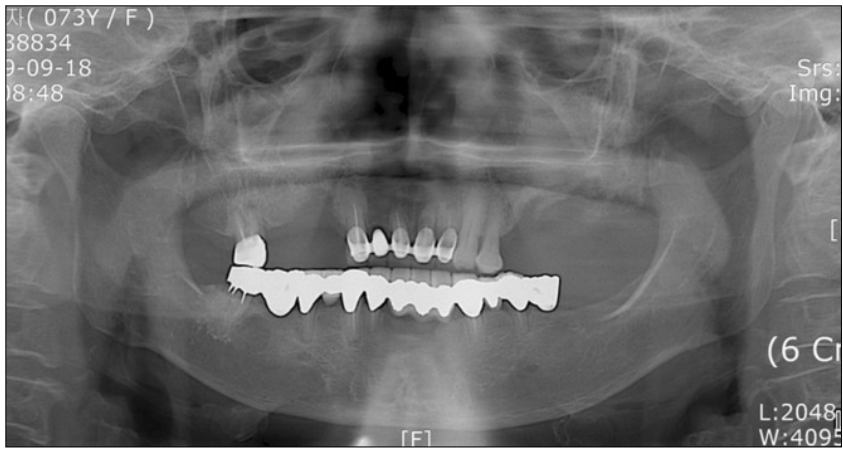

Fig. 5. After 3 months from the initial visit, the inflammatory state has been clinically relieved and according to the panoramic view taken for preoperative examination purpose, significant bone formation and sequestrum (maxillary left molar and premolar area) formation can be identified.

discharged on October 7th, 2009. The result of the biopsy on October 12th, 2009 indicated acute, chronic osteomyelitis and conclusively, BRONJ.

Ambulatory treatment was performed after discharge until October 29th, 2009 and the patient was educated to maintain a good oral hygiene status.

Four weeks after surgery on November 5th, 2009, panorama examination was performed (Fig. 7) and on November 20th, 2009, the operation area seemed to be in satisfactory state.(Fig. 8) After 10 weeks, on December 18th, 2009, prominent bone formation was observed in the radiographic images.(Fig. 9) Periodic evaluation will be continued. 

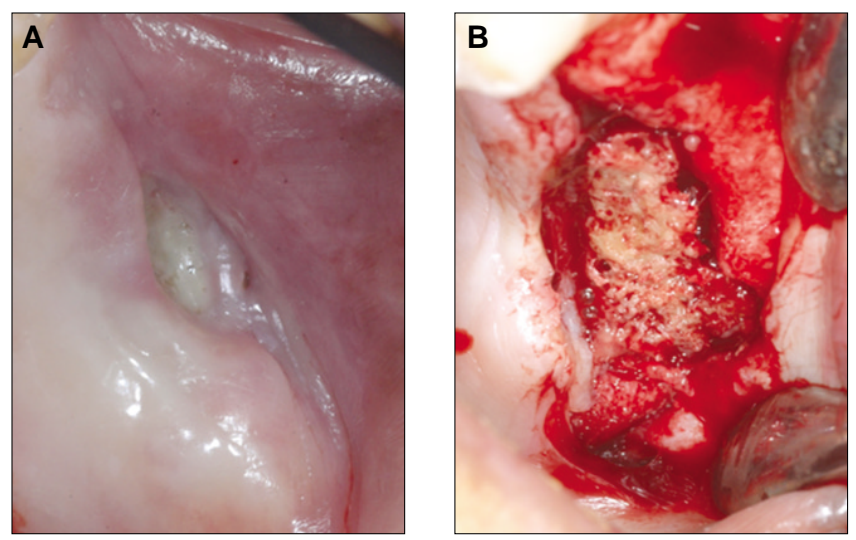

Fig. 6. A. Left Maxillary Lesion, B. The Sequestrum was removed, and the tissue removed was further examined through biopsy for a confirmed diagnosis.

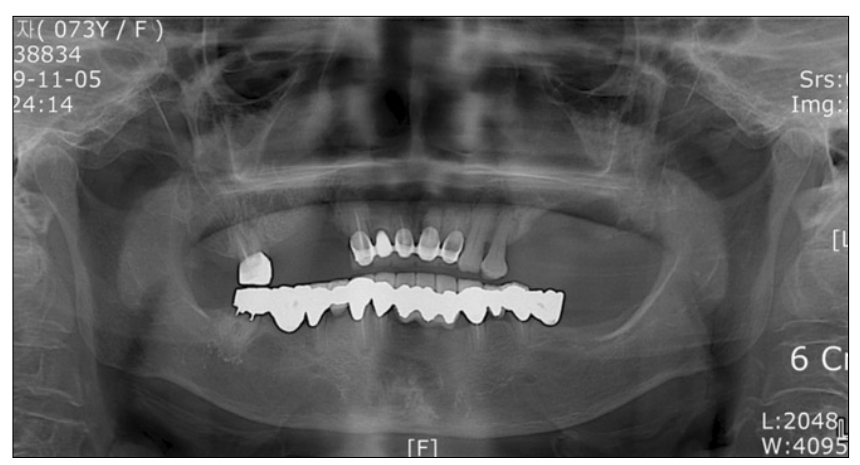

Fig. 7. Four weeks after surgery, according to the radiographic image the irregularity of the maxillary left and right surgery area has decreased and, due to new bone formation, was merging with the adjacent bone.

\section{Discussion}

\section{Definition of BRONJ}

To be diagnosed as BRONJ, all of the three below should be satisfied ${ }^{6}$.

- Bone exposed in jaw lasting at least 8 weeks in spite of the proper treatment.

- Had taken BPs or has been taking BPs.

- No history of radiation therapy on the jaw.

\section{Epidemiology of BRONJ}

- Though partly it occurs naturally, BRONJ is usually related to the tooth extraction and oral surgery.

- The longer BPs taken, the higher the risk.

- Oral BPs has lower risk of BRONJ than intravenous BPs.

- There's no difference in incidence of BRONJ between oral BPs and intravenous BPs at the dose for osteoporosis treatment.
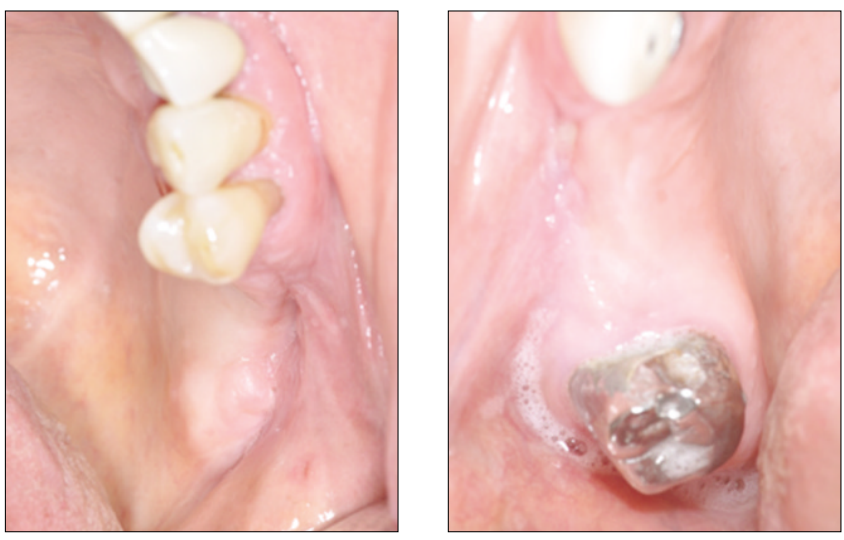

Fig. 8. Six weeks after surgery, the surgery area of the maxillary left and right side is healing progressively.

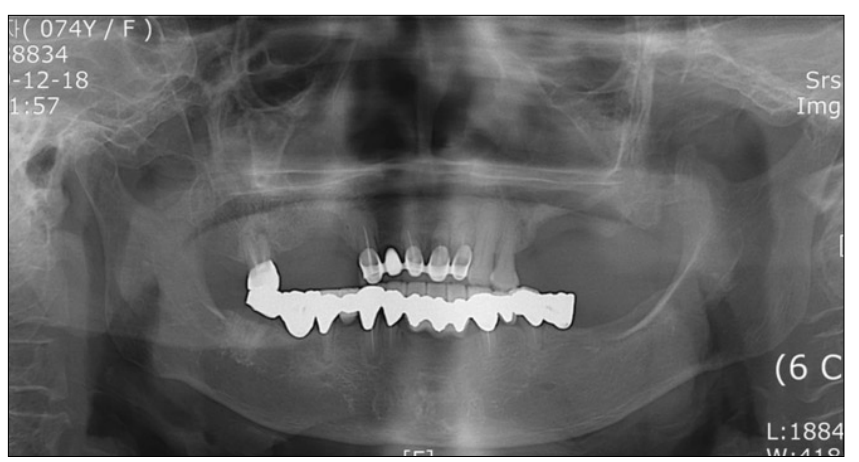

Fig. 9. Ten weeks after surgery, according to radiographic image, the bone of the surgery area in the maxillary left and right side is maturing into similar configuration as the adjacent bone.

- Though oral BPs has lower risk of BRONJ, the long-term (more than 3 years) administration of it increases the risk.

- If patient has other disease or takes steroids at the same time, the risk of BRONJ increases despite the short-term administration.

- It seems that the oral BPs has higher risk of BRONJ than reported before?

\section{Background about Bisphosphonates and BRONJ}

BPs has been used to decrease osteoclasis and enhance the osteogenesis since Francis et al. ${ }^{8}$ reported that diphosphonates suppress the dissolution of hydroxyapatite in vitro and decrease the bone absorption in vivo. Etidronate and clondronate which is first generation of BPs don't have nitrogen in their structure. The Food and Drug Administration (FDA) didn' $t$ approve them as medicine for osteoporosis. Second generation BPs contains nitrogen in structure and has high affinity to bone, so the suppression effect on osteoclastic activities is improved than first generation BPs. Pamidronate which 
is an intravenous BPs was approved as medicine for multiple myeloma, osteoclastic metastasis in breast cancer and Paget's disease by FDA in 1995. In the same year alendronate was approved as medicine for osteoporosis after menopause and osteoporosis arising from corticosteroids.

Then zoledronic acid which is an intravenous BPs got approved by FDA as medicine for multiple myeloma and bone metastatic cancer.(Table 1)

BPs (nBPs), having a structure including nitrogen, is known to show effectiveness as reducing farnesyl diphosphate (FPP) synthase which is one of the mevalonate pathways in osteoblast $^{9}$.(Fig. 10)

These reducing results are originated from different mechanisms according to concentration of $\mathrm{nBPs}^{10}$.

At first, when the concentration of nBPs is low, small GTPase' s isoprenylation (essential step for cytoskeleton, vesicular trafficking and membrane ruffling in osteoblast) like

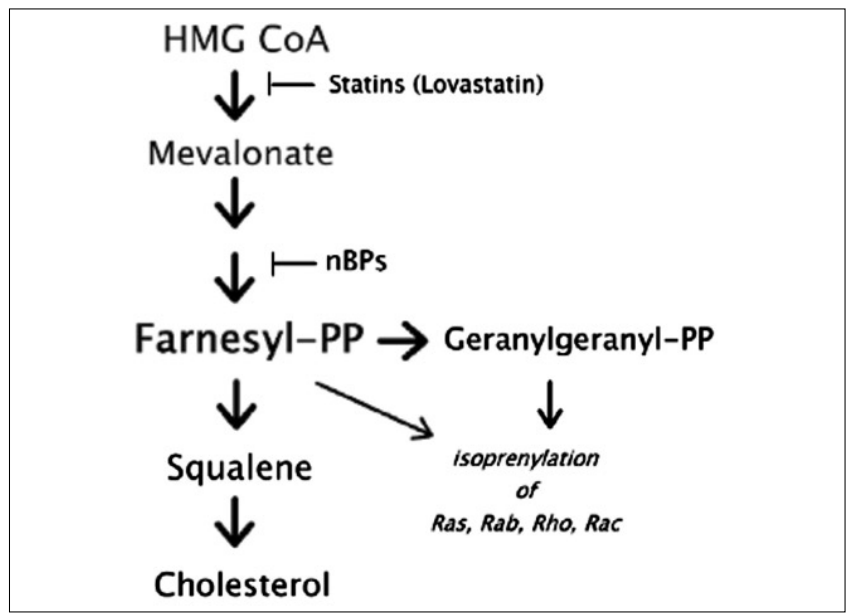

Fig. 10. Nitrogen-containing bisphosphonates inhibit farnesyl diphosphate (FPP) synthase, an enzyme in mevalonate pathway. FPP synthase is responsible for isoprenylation of small GTPases that promote an array of activities in the osteoclasts that control bone resorption. Without this activity, bone resorption is slowed.

(HMG: 3-hydroxy-3-methylglutaryl)
Ras is hindered by the reduction of nBPs FPP synthase. As well known, osteoclasts attach to the surface of bone by integrin, then plasma membrane of osteoclasts and the attached surface of bone form sealing zone. At this time, osteoblasts transform their plasma membrane to ruffled membrane to have extended surface in the sealing zone. Osteoclasts turn the sealing zone to acidic environment by releasing $\mathrm{H}+$ into the sealing zone through proton pump in ostelclasts' membrane enabling a decalcification of bone surface in the sealing zone. Furthermore, cathepsin $\mathrm{K}$, having a role in bone resorption, from inner vesicles of osteoclasts is transferred to the sealing zone, and start to decompose type 1 collagens caused from decalcified bone ${ }^{11}$. If isoprenylation is reduced by nBPs, these bone resorption stages by osteoclasts will not occur easily.

On seconds, when the concentration of nBPs is high, nBPs reduces the geranylgeranylation of Rho and Rac, eventually leading to repression of differentiation in osteoclasts.

Finally, when the concentration of nBPs is about $100 \mu \mathrm{M}$, the apoptosis of osteoclasts is induced by Rho and Rac.

BPs which are currently being marked are containing same original structure. They are distinguished by only substituent group (-R group).(Fig. 11) Referred reducing potency of FPP synthase of nBPs show differences by five hundred times according to the kind of substituent groups containing nitrogen ${ }^{10}$.

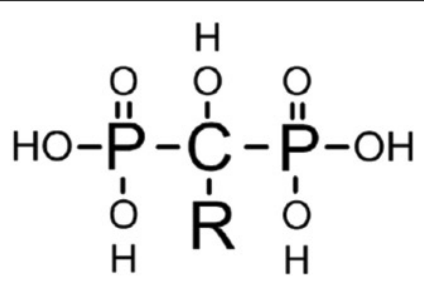

Fig. 11. Bisphosphonates used most frequently in the clinic today have a characteristic structure. All have a hydroxyl group on the carbon atom that confers high affinity for calcium and the skeleton. They vary only at the R-group, which always contains a nitrogen atom that is in either an alkyl or a heterocyclic structure.

Table 1. Amino bisphosphonate drugs ${ }^{12,13}$

\begin{tabular}{ccccc}
\hline Drug name & Active ingredients & Dosage form: route & FDA approval & Relative potency $^{1}$ \\
\hline Fosamax & Alendronate sodium & Tablet: oral & 1995 & 1,000 \\
Actonel & Risedronate sodium & Tablet: oral & 1998 & 5,000 \\
Boniva & Ibandronate sodium & Tablet / injectable: & 2003 & 10,000 \\
& & oral / IV injection & 2006 & 1991 \\
Aredia & Pamidronate disodium & Injectable: IV infusion & 2001 & 100 \\
Zometa & Zoledronic acid & Injectable: IV infusion & 2007 & 100,000 \\
Reclast & Zoledronic acid & Injectable: IV infusion & 100,000
\end{tabular}

$\left({ }^{1}\right.$ : relative to etidronate, a non-nitrogen-containing bisphosphonate with relative potency of 1, FDA: the Food and Drug Administration) 
Traditionally, the indications of BPs are osteoporosis, hypercalcemia of malignancy, multiple myeloma, breast cancer metastatic disease to bone and prostate cancer ${ }^{14}$. Recently, BPs is used as medicine or supplements for better effectiveness on the following disease such as giant cell lesions of the jaws, osteogenesis imperfect, fibrous dysplasia, Gaucher' s disease, osteomyelitis, and orthopedic implants, etc. ${ }^{15}$.

\section{Hypotheses about the cause of BRONJ}

1) Pathogenesis of BRONJ-remodeling suppression

The mechanism of the origin of BRONJ is not yet clearly unveiled, but some hypotheses are suggested. The most generally accepted theory of them says that bone remodeling suppression induced from BPs is the cause. Premises supporting this theory are 1) Jaw bones have high remodeling rate than other bones. 2) BPs suppress bone remodeling ${ }^{16,17}$.

In case of human, the bone remodeling rate of cortical bone of jaw is 10-20 times faster than that of iliac bone ${ }^{18,19}$. Namely, unusual osteonecrosis occurs at jaw due to the rapid bone remodeling of jaw and suppression of remodeling by BPs. Allen et $a l .{ }^{20}$ reported the effects of BPs on bone remodeling at cortical bone of mandible. In this experiment, beagles were put into two groups. The general dosage of alendronate for osteoporosis treatment was orally administrated to one group for three years while the other was treated with 5 times more dosage. A histological evaluation of bone remodeling at cortical bone of beagles was performed in comparison to a control group that wasn' $t$ treated with alendronate. In case of the control group, the bone remodeling rate at cortical bone of mandible was 10 times higher than that of long bone, with maximum remodeling rate at alveolar bone. On the other hand, the beagle groups that were daily administrated with alendronate showed a significant decrease of remodeling rate at cortical bone of mandible with maximum decrease at alveolar bone.

The BRONJ rate of cancer patients treated with BPs was considerably higher than osteoporosis patients treated with BPs, which was due to the difference in the degree of bone remodeling suppression. The BPs for cancer patients had higher potency and binding affinity (zoledronate and pamidronate) than that was prescribed for osteoporosis patients, and also administrated more frequent and in higher dosage ${ }^{16}$.

Among non BPs series drugs that affect bone metabolism, calcitonin and selective estrogen receptor modulators (SERMs) aren' $t$ reported to develop BRONJ, because those drugs aren' $t$ assumed to have over $50 \%$ turnover suppression ${ }^{16}$.

But the fact that denosumab, a drug recently undergone clini- cal trials for osteoporosis and cancer treatment which decreases bone remodeling on a similar level to BPs, does not develop BRONJ even if it is administrated in the same dosage, times and period as BPs, weakens the hypothesis above ${ }^{21}$.

Many efforts including stop taking BPs, debridement and antibacterial washes had no curative effects on the BRONJ patients, but when they were treated with tetriparatide (recombinant human parathyroid hormone) which enhances bone remodeling, BRONJ was healed ${ }^{22-24}$.

There are quite many cases of developing BRONJ after tooth extraction in the patients treated with BPs over long period. For these patients, the formation of woven bone during healing process of extraction wound is normal but the process of bone remodeling from callus to lamellar bone is delayed and this seems to be the reason for BRONJ ${ }^{25,26}$.

\section{2) Pathogenesis of BRONJ - focus on the osteocyte}

We have discussed about the direct or indirect evidences that BRONJ is occurred by BPs which suppress bone remodeling. But why does the suppression of bone remodeling lead to osteonecrosis of jaw? Explanations to this question are primarily focused on osteocytes. First, though osteocyte is known as a long living cell, it also reaches natural cell death after a certain period of time. In ordinary condition, dead osteocytes are replaced by new ones when existing bone tissues are turned into new bones. However, this replacement is inhibited when bone remodeling is suppressed by BPs and the lacunae where osteocytes sited are left as hollow cavities. These cavities can be identified by local mineralization through fuchsin dye. Osteocytes are connected to each other by intracanaliculi and they play an important role as a source of nutriment to bones. Therefore the bones with dead osteocytes will easily be necrotized $^{27}$.

Second hypothesis is that BPs that are sticked inside the bones, especially at lacunae of osteocytes, act as toxic substances to osteocytes and lead these osteocytes to cell death which eventually develop BRONJ ${ }^{28}$.

3) Pathogenesis of BRONJ - the antiangiogenic effects of BPs

It is widely admitted that the cause of osteoradionecrosis is due to the avascular necrosis induced by radiation. A hypothesis analogized by this fact is that abnormal angiogenesis takes place by BPs and develop BRONJ ${ }^{16}$. Many studies on this antiangiogenic property of BPs are in progress to prove this hypothesis. In fact, BPs has been used as anticancer treatment in expectation of the potential effect to suppress angiogenesis in the growth process of malignant tumor ${ }^{29,30}$. Furthermore, the 
antiangiogenic property of BPs is proved by many experiments. However, most of these experiments were performed in vitro, and few of them had in vivo results ${ }^{16}$. Through in vitro and rat experiments, pamidronate and zoledronate are reported to have suppression effect to capillary regeneration, epithelial growth factor and angiogenesis ${ }^{2,31}$. Suppression to mevalonate pathway induced by BPs inhibits guanosine triphosphate (GTP)-signaling pathway which is involved in epithelial migration ${ }^{32}$. Woods and colleagues have proved through both in vitro and in vivo experiments that zoledronate suppresses angiogenesis in proportion to the dosage ${ }^{32}$. However, drugs like thalidomide are evidences against this hypothesis above, in the respect that they do not develop BRONJ even if they have more antiangiogenic effect than $\mathrm{BPs}^{2}$.

In many cases, BRONJ are developed after invasive dental treatments, especially tooth extraction. Looking into the healing process of extraction wound, blood clot is formed in the wound in the early stage, which is transformed into granulation tissue after certain time. Afterward, connective tissue and preosseous tissue are formed, and eventually the extraction wound is filled with new generated bone. The whole healing process will encounter problems even if one of the stages goes wrong. According to the hypothesis above, the extraction wound healing process of angiogenically suppressed patients due to a long term administrated BPs, won' t go along smoothly. Furthermore, osteoclast suppression by BPs will induce inhibition of bone remodeling at extraction wound, which will delay healing process and lead to BRONJ ${ }^{16}$.

In its recently announced two case reports, similar bone exposure of mandible as in BRONJ has been reported on cancer patients taking bevacizumab instead of BPs, even though they didn' $t$ receive dental treatments including extraction or surgery $^{33}$. Despite these evidences that vascular structure deformation is related with BRONJ development, the fact is that we are still in lack of systematic research results.

It is also suggested that altered bloodstream caused by BPs will effect development of BRONJ ${ }^{34}$. As metabolic activity of a tissue is proportional to the bloodstream of that tissue, the jaw bone where the bone remodeling is quite active has rich bloodstream. Patients treated with BPs will have reduced jaw bone remodeling due to BPs and the bloodstream provided to jaw will decrease.

They insist that, when high blood flow is needed after extraction or infection, the changed-vessel is not enough then it can cause osteo-necrosis ${ }^{35,36}$.

4) Pathogenesis of BRONJ-infection

It is not clear whether infection is the immediate cause or it is secondary phenomenon. Phenomenalizing, actinomyces is the most common cell in pathologic tissue of BRONJ. Not related with osteoclast, bacteria or fibroblast-like cell can lysis bone by releasing acid or protease. Normal bone remodeling process occurs by coupling of osteoclast and osteoblast. That is, they activate each other. So, the osteolysis non-related with osteoclast inhibits induction of osteogenesis by osteoblast ${ }^{37,38}$. But, this hypothesis is an insubstantial reason for causing BRONJ.

\section{5) Other Hypotheses of BRONJ}

Few reported that diabetes ${ }^{39}$, smoking, obesity ${ }^{40}$, tooth extraction $^{3}$ and taking steroids ${ }^{3}$ can be reason for BRONJ, however they are regarded as co-factors.

\section{Incidence frequency and risk factor of BRONJ}

If high dose of BPs used to malignant tumor patients, incidence frequency of BRONJ is increased. Statistically, incidence frequency is $0.8-12 \%$.

Oral inoculation of BPs for treatment of osteoporosis causes BRONJ less than injection of BPs for anticancer treatment.

Depending on the statistics of manufacture of alendronate, in case of oral oculation, incidence frequency of BRONJ is 0.7/100,000 person/year of exposure.

Risk factors of BRONJ are drug-related factor, local factor, demographic factor, systemic factor, genetic factor and preventive factor.

1) Drug-related factor

(1) Potency of BPs: zoledronate>pamidronate>oral bisphonate ${ }^{41-44}$.

Incidence rate of BRONJ of cancer patient didn't have intravenous (IV) BPs is 2.7-4.2 times more higher than that of cancer patient had IV $\mathrm{BPS}^{43}$.

(2) Period of treatment: The longer have treatment, the higher incidence rate is ${ }^{41,42}$.

2) Local factor

(1) Dental surgery: tooth-extraction, Implantation surgery, peri-apical surgery, perio-surgery including bone $\operatorname{loss}^{42-44}$. Incidence rate of BRONJ of had dental surgery patient is 5-21 times more than that of non-dental surgery patient ${ }^{43,44}$.

(2) Anatomical factor

Mandible:maxilla=2:1, thin mucosa covering protrusion, poor intra-oral hygiene, drinking and smoking?

(3) Co-existing intra oral disease: of IV BPs treatment 
patients, it shows 7 times more incidence rate of BRONJ if patient have inflammatory dental disease.

\section{3) Demographic factor}

The whites has higher incidence rate than the blacks ${ }^{44}$.

For aging, malignancy, chronic renal insufficiency, diabetes, anti-cancer therapy, taking steroids, incidence rate is increased?

\section{4) Genetic factor}

According to Sarasquete et al. ${ }^{45}$ about patients treated with IV BPs, occurrence risk for BRONJ is increased due to genetic difference (cytochrome P450-2c [CYP2C8] monobase pleomorphism of gene).

\section{5) Preventive factor}

It is recommended dental treatments are proceeded before IV BPs treatment, if they are needed?

\section{6) Co-factor}

Occurrence risk for BRONJ is increased for the reasons of corticosteroid treatment, diabetes, poor intra-oral hygiene, drinking, smoking and anti-cancer therapy?

According to Krane ${ }^{46}$, glucocorticoid inhibits transcription of collagenase.

It attenuates degradation of type 1 collagen.

Osteocyte or osteoblast adheres to the site where type 1 collagen degradation occurs. If not, apoptosis happens. Osteonecrosis of the jaw $(\mathrm{ONJ})$ is found in patients who are treated with glucocorticoid. Observing the tissue, cavity caused by death of osteocyte is filled with minerals. It is very similar to histologic findings of $\mathrm{BRONJ}^{46}$. If patients, who have been treated with BPs for a long time, and prescribed glucocorticoid (it is used for cancer treatment), it can be inferred that, the risk incidence of BRONJ is increased.

\section{Clinical manifestation of BRONJ}

Non-vitally exposed bone is a clinical manifestation of $\mathrm{BRONJ}^{47}$. Patients complain about severe pain and discomfort ${ }^{3}$. In most cases, BRONJ occurs after bone damage like tooth extraction, however some occur spontaneously ${ }^{47}$. Even it commonly occurs in mandible, it also occurs in maxilla ${ }^{31}$ (mandible:maxilla=2:1). While the disease proceeds, inflammatory reaction and infection can be observed, it is a typical symptom of BRONJ. The classification by clinical stages which Ruggiero et al.$^{48}$ suggested in AAOMS in 2009 follows as Table 2 .
Table 2. Clinical stages of BRONJ patient ${ }^{12}$

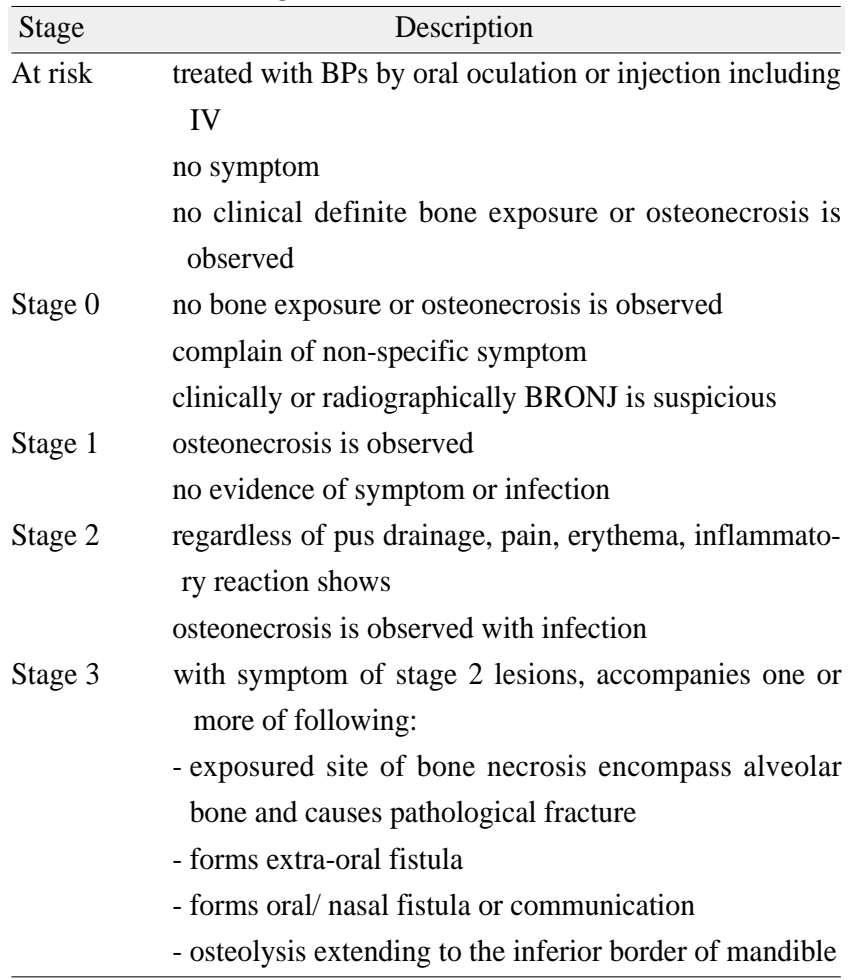

Diseases differentially diagnosed from BRONJ are alveolar osteitis, sinusitis, gingivitis, periodontitis, caries, periapical pathology, temporomandibular joint (TMJ) disorders and so on.

\section{Radiographic findings of BRONJ}

Through periapical view, panorama, CT, and magnetic resonance imaging (MRI), any radiographic change is rare in the bone of early developing stage of BRONJ. Even nBPs supresses osteoclasts and bone mineral density (BMD) goes up, an increase of radiopacity develops systemically and uniformly throughout entire maxillary bone, discrimination of any radiographic is difficult.

When osteolysis develops, indurative or worm-eaten appearance surrounded by radiopaque band, 'sequestrum' is detected ${ }^{49}$.

Additional radiographic findings of BRONJ are,

1) A tooth extraction site with unfilled by bone after considerable time.

2) Remaining lamina dura on a tooth extraction site is indurated and prominent.

Great attention should be paid to these two radiographic findings, because they precede clinical symptoms ${ }^{12}$. When 
inflammatory change or infection is developed in the soft tissue which covers lesions, the change is able to be detected by typical dental radiographics, CT, and MRI. Increased uptake of positron emission tomography (PET) on the patient with BRONJ has been reported ${ }^{50}$.

\section{Biochemical markers applicable in establishing BRONJ}

Bone metabolism related biochemical markers can be categorized into two groups. 1) Bone formation related markers. 2) Bone resorption related markers

Bone formation related markers that can be obtained from serum are bone specific alkaline phosphatase (BSALP), osteocalcin (OC), carboxyterminal propeptide of type I collagen (PICP), aminoterminal propeptide type I collagen (PINP) and so on ${ }^{13}$.

Bone resorption related markers that can be obtain from urine are free and total pyridinoline (PYR), free and total deoxypyridinoline (DPD), N-telopeptide of collagen crosslinks (NTX), C-telopeptide of cross-links (CTX) and so on. Those can be obtained from serum are NTX and CTX abovementioned $^{13}$.

Apart from above, bone turnover related markers are serum calcium, serum parathyroid hormone, serum 1, 25-dihydroxyvitamine, serum osteoprotegrin, serum osoteopontin, serum procollagen type I $\mathrm{N}$ propeptide, receptor activator of nuclear factor $\kappa \beta$ ligand $(\mathrm{NF} \kappa \beta)$ and so on ${ }^{13}$.

Among these, recently CTX marker received wide attention. By measuring serum CTX value, patient's BRONJ developing resk can be assessed.(Marx et al..$^{51}$ ) This telopeptide segment is formed while the bone resoprtion process when osteoclast cut the cross link of collagen. Therefore the serum CTX value is directly proportional to the osteoclast activity. The CTX value of a patient with osteoporosis who doesn' $t$ take BPs is 400 $\mathrm{pg} / \mathrm{mL}$ while who takes BPs is $150 \mathrm{pg} / \mathrm{mL}$. On recent paper, Kunchur et al..$^{52}$ suggested the guideline for prevention of BRONJ and management of BRONJ patient using CTX test. A total of 348 patients underwent a fasted morning CTX test. Of these, 222 were patients at risk of ONJ who had been referred for extractions, 15 had ONJ, and 113 were controls. The 215 patients taking long-term oral bisphosphonates were older (71+/-11.6 years), were predominantly women with osteoporosis, and were medically compromised. The average CTX value was $238+/-144 \mathrm{pg} / \mathrm{mL}$, with 98 having a value less than 200 $\mathrm{pg} / \mathrm{mL}$. One patient with a CTX value of $126 \mathrm{pg} / \mathrm{mL}$ developed ONJ after an extraction. Seven intravenous bisphosphonate patients underwent extractions with no cases of ONJ developing. The CTX value was $329+/-354 \mathrm{pg} / \mathrm{mL}$, with 4 less than
$200 \mathrm{pg} / \mathrm{mL}$. Fifteen patients developed ONJ, 12 after extractions and 3 spontaneously. Of these, 7, who were still taking a bisphosphonate at presentation, had a CTX value of 116 $\mathrm{pg} / \mathrm{mL}$. The CTX test is not predictive of the development of BRONJ but the test is useful to identify patients in "riskzone", which is a value of less than $150 \mathrm{pg} / \mathrm{mL}$ to $200 \mathrm{pg} / \mathrm{mL}^{52}$.

With these bone formation related markers, especially CTX, patients with $\mathrm{nBP}$ treatment can be 1) assessed for the medicinal effect of prescribed nBP 2) assessed for the risk of BRONJ 3) assessed for the stage of BRONJ when they are established BRONJ patients. Yet it is debatable, when supported with theoretical evidence, biochemical markers can be a useful evaluation method ${ }^{13}$.

\section{Prevention and teatment of BRONJ}

The best treatment of BRONJ is prevention. That is because even patient dicontinuate uptaking of BPs, the effect of discontinuation is subtle due to the characteristics of BPs that it remains in the bone for several years. Furthermore when ostectomy on the lesion site is performed, the procedure itself also considered as an invasive factor hence deteriorate the symptom.

For the prevention of BRONJ, following patient education is recommended.

1) When dental treatment is needed, patient notify dental clinicians of their bisphosphonate uptake

2) Prior to the BPs treatment, notify patients of advantages of BPs and possibility of developing BRONJ as a rare complication.

3) Patients need to maintain good oral hygiene.

4) Notify clinicians when patient has risk factors or syptoms of BRONJ

5) Notify clinicians when patient has edema, pain or exposed bone ${ }^{7}$.

Prior to treatment with BPs, any unsalvaged teeth should be removed, all invasive dental procedures should completed and optimal oral health should be achieved.

Patient with established BRONJ should be treated by skillful dental specialists, and the purpose of treatment should to release pain and infection of soft tissue and bone, and minimize osteonecrosis of the jaw. Chlorhexidine mouthwash, an antiseptic agent, is an effective treatment method for infection, and if there is any evidence for infection, systemic antibiotics are recommended. The conservative treatment is the first choice because there is a possibility that periodontal surgery makes the surgical site re-necrotized, thus it should be delayed as long as possible. It should be done in the case of no less 
than stage 3 or when necrotized bone can be separated clearly. There is a chance that the necrotized bone and sharp bones can stimulate soft tissue around them, so just a part of bone is resected without giving any harm to surrounding bone. When it comes with a vast range of bone necrosis or pathologic fracture, partial mandibulectomy could be necessary?

Treatment strategy (recommended by AAOMS in 2009) according to the disease stage is like below.

1) At risk: special treatment is not required for the patients having BPs treatment and being in risk of progressing to BRONJ. However, the possibility of BRONJ and the symptoms of this disease should be explained to the patients.

2) Stage 0: the treatment and administration of antibiotic (if necessary) are needed. Conservative treatment is used for the patient with dental caries and periodontal problems.

3) Stage 1: chlorhexidine gluconate $0.12 \%$, an antiseptic agent, is an effective treatment for the patients of this stage. Surgery is not required.

4) Stage 2: administration of oral antibiotics and chlorhexidine mouthwash is used. Most of the cases, penicillin works, however, if patients are allergic to it, quinolone, metronidazole, clindamycin, doxycycline, and erythromycin can be administrated. If there is no response to oral antibiotics, combination antibiotic therapy or injection is used. Necrotized tissue is removed superficially without giving stimulus to the soft tissue.

5) Stage 3: oral antiseptic mouthwash is used. Releasing pain and systemic antibiotic administration is required. Necrotized bone is removed.

Recommendations for quitting BPs administration are as follows 1) BPs administration is stopped for the BRONJ patient

2) Generally if the boundary of necrotized site can be wellformed (that is, sequestrum is formed) in 6 to 12 months of interruption, it can be healed well by surgical resection.

3) While systemic condition is satisfactory, alternative medicine administration can be considered.

\section{Implant and BRONJ}

There is a discussion when implants are placed to the patient who are prescribed BPs for a long period, it can be a factor of BRONJ. According to Wang et al. ${ }^{24}$, clinical symptom of bone necrosis after normal dental implant placing is found in the patients who are administrated BPs more than 10 years. Kos et $a l .{ }^{53}$ showed the result of 34 patients having BRONJ treatment due to multiple myeloma, breast cancer, prostatic cancer, and osteoporosis. For 31 patients, BRONJ happened by tooth extraction, apicoectomy, ill-fitted denture, cyst enuncleation, implant pacing and trauma. Madrid et al..$^{54}$ announced the result analyzing one prospective study and three retrospective series (using 271 patients). Following this study, patients who administrated oral BPs less than 5 years didn' t show BRONJ after implant placement, thus short-termed oral BPs administration is not a contra-indication for the implant placement. Moreover, 1 to 4 years' oral BP administration didn' $t$ affect the short-term implant survival rate.

Reports, so far, has shown that in the case of no longer than 3 to 5 years' oral BPs administration, implant placement is not a risk factor for BRONJ. To reach the exact conclusion, longterm follow-up studies and systemic studies are needed.

Marx et al..$^{51}$ suggested that CTX test should be practiced before implant treatment for the patients who have had oral BPs more than 3 years, even though less than 3 years, are prescribed for corticosteroid, and got anti-cancer treatment. According to this protocol, When CTX level is less than 150 $\mathrm{gp} / \mathrm{mL}$, drug holiday and delayed surgery are recommended. To apply this result to the clinical case, more studies are necessary.

\section{IV . Conclusion}

Recently reported BRONJ has been studied using domestic and international reports, and the cause, mechanism, risk factor of BRONJ are analyzed. Furthermore, clinical symptom, radiographic features, histopathologic findings, physicochemical tests including biochemical marker of BRONJ, which are needed to diagnosis are explained. The methods of prevention and treatment of BRONJ are discussed based on lots of references and clinical experience. To improve clinicians' understanding, cases that the author has experienced are introduced and for them to have more confidence in seeing these kind of patients is anticipated. More effective treatment and prevention methods such as development medicine of less risky of BRONJ, quick diagnosis, short treatment period treatment is looked forward to for the healthier lives of the people.

\section{References}

1. Marx RE. Pamidronate (Aredia) and zoledronate (Zometa) induced avascular necrosis of the jaws: a growing epidemic. J Oral Maxillofac Surg 2003;61:1115-7.

2. Marx RE, Sawatari Y, Fortin M, Broumand V. Biphosphonateinduced exposed bone (osteonecrosis/ostoepetrosis) of the jaws: risk factors, recognition, prevention, and treatment. J Oral Maxillofac Surg 2005;63:1567-75.

3. Advisory Task Force on Bisphosphonate-Related Ostenonecrosis of the Jaws, American Association of Oral and Maxillofacial Surgeons. American Association of Oral and Maxillofacial 
Surgeons position paper on bisphosphonate-related osteonecrosis of the jaws. J Oral Maxillofac Surg 2007;65:369-76.

4. Kwon YD, Yoon BW, Walter C. Bisphosphonate, is it an emerging risk factor in oral surgery? J Korean Assoc Maxillofac Plast Reconstr Surg 2007;29:456-62.

5. Park YD, Kim YR, Kim DY, Chung YS, Lee JK, Kim YG, et al. Awareness of Korean dentists on bisphosphonate related osteonecrosis of the jaws: preliminary report. J Korean Assoc Oral Maxillofac Surg 2009;35:153-7.

6. Ruggiero SL, Dodson TB, Assael LA, Landesberg R, Marx RE, Mehrotra B; American Association of Oral and Maxillofacial Surgeons. American Association of Oral and Maxillofacial Surgeons position paper on bisphosphonate-related osteonecrosis of the jaws-2009 update. J Oral Maxillofac Surg 2009;67(5 Suppl):2-12.

7. The Korean Endocrine Society, the Korean Society of Bone Metabolism, the Korean Society of Osteoporosis, the Korean Association of Oral and Maxillofacial Surgeons. Bisphosphonate related osteonecrosis of the jaw (BRONJ): position statement of Korea. J Korean Endocr Soc 2009;24:227-30.

8. Francis MD, Russell RG, Fleisch H. Diphosphonate inhibit formation of calcium phosphate crystals in vitro and pathological calcifications in vivo. Science 1969;165:1264-6.

9. van Beek E, Pieterman E, Cohen L, Löwik C, Papapoulos S. Farnesyl pyrophosphate synthase is the molecular target of nitrogen containing bisphosphonates. Biochem Biophys Res Commun 1999;264:108-11

10. Kimmel DB. Mechanism of action, pharmacokinetic and pharmacodynamic profile, and clinical applications of nitrogen-containing bisphosphonates. J Dent Res 2007;86:1022-33.

11. Väänänen HK, Zhao H, Mulari M, Halleen JM. The cell biology of osteoclast function. J Cell Sci 2000;113:377-81.

12. Fantasia JE. Bisphosphonates-what the dentist needs to know: practical consideration. J Oral Maxillofac Surg 2009;67(5 Suppl):53-60

13. Hillner BE, Ingle JN, Berenson JR, Janjan NA, Albain KS, Lipton A, et al. American Society of Clinical Oncology guideline on the role of bisphosphonates in breast cancer. American Society of Clinical Oncology Bisphosphonates Expert Panel. J Clin Oncol 2000;18:1378-91.

14. Mehrotra B. Bisphosphonates-role in cancer therapies. J Oral Maxillofac Surg 2009;67(5 Suppl):19-26.

15. Landesberg R, Eisig S, Fennoy I, Siris E. alternative indications for bisphosphonate therapy. J Oral Maxillofac Surg 2009;67(5 Suppl):27-34

16. Allen MR, Burr DB. The pathogenesis of bisphosphonate-related osteonecrosis of the jaw: so many hypotheses, so few data. J Oral Maxillofac Surg 2009;67(5 Suppl):61-70.

17. Russell RG, Xia Z, Dunford JE, Oppermann U, Kwaasi A, Hulley PA, et al. Bisphosphonates: an update on mechanisms of action and how these relate to clinical efficacy. Ann N Y Acad Sci 2007;1117:209-57.

18. Garetto LP, Chen J, Parr JA, Roberts WE. Remodeling dynamics of bone supporting rigidly fixed titanium implants: a histomorphometric comparison in four species including humans. Implant Dent 1995;4:235-43

19. Han ZH, Palnitkar S, Rao DS, Nelson D, Parfitt AM. Effects of ethnicity and age or menopause on the remodeling and turnover of iliac bone: implications for mechanism of bone loss. J Bone Miner Res 1997;12:498-508.

20. Allen MR, Burr DB. Mandible matrix necrosis in beagle dogs after 3 years of daily oral bisphosphonate treatment. J Oral Maxillofac Surg 2008;66:987-94.

21. Lewiecki EM, Miller PD, McClung MR, Cohen SB, Bolognese MA, Liu Y, et al. Two-year treatment with denosumab (AMG 162) in a randomized phase 2 study of postmenopausal women with low BMD. J Bone Miner Res 2007;22:1832-41.

22. Harper RP, Fung E. Resolution of bisphosphonate-associated os- teonecosis of the mandible: possible application for intermittent low-dose parathyroid hormone [rhPTH(1-34)]. J Oral Maxillofac Surg 2007;65:573-80

23. Lau AN, Ali SH, Adachi JD. Resolution of osteonecosis of the jaw after teriparatide [recombinant human PTH (1-34)] therapy. Osteoporos Int 2008;19 Suppl 1:S80.

24. Wang HL, Weber D, McCauley LK. Effect of long-term oral bisphosphonates on implant wound healing: literature review and a case report. J Periodontol 2007;78:584-94.

25. Li C, Mori S, Li J, Kaji Y, Akiyama T, Kawanishi J, et al. Longterm effect of incadronate disodium (YM-175) on fracture healing of femoral shaft in growing rats. J Bone Miner Res 2001; 16:429-36.

26. Peter CP, Cook WO, Nunamaker DM, Provost MT, Seedor JG, Rodan GA. Effect of alendronate on fracture healing and bone remodeling in dogs. J Orthop Res 1996;14:74-9.

27. Frost HM. In vivo osteocyte death. J Bone Joint Surg Am 1960; 42A:138-43.

28. Roelofs AJ, Coxon FP, Ebetino FH, Bala JF, Kashemirov BA, McKenna CE, et al. Use of a fluorescent analogue of risedronate to study localisation and cellular uptake of bisphosphonates in vivo. Bone 2008;42 Suppl 1:S85.

29. Guise TA. Antitumor effects of bisphosphonates: promising preclinical evidence. Cancer Treat Rev 2008;34 Suppl 1:S19-24.

30. Lipton A. Emerging role of bisphosphonates in the clinic-antitumor activity and prevention of metastasis to bone. Cancer Treat Rev 2008;34 Suppl 1:S25-30.

31. Ruggiero SL, Mehrotra B, Rosenberg TJ, Engroff SL. Osteonecrosis of the jaws associated with the use of bisphosphonates: a review of 63 cases. J Oral Maxillofac Surg 2004;62:52734.

32. Wood J, Bonjean K, Ruetz S, Bellahcène A, Devy L, Foidart JM, et al. Novel antiangiogenic effects of the bisphosphonate compound zoledronic acid. J Pharmacol Exp Ther 2002;302:1055-61.

33. Estilo CL, Fornier M, Farooki A, Carlson D, Bohle G 3rd, Huryn JM. Osteonecrosis of the jaw related to bevacizumab. J Clin Oncol 2008;26:4037-8.

34. Somerman MJ, McCauley LK. Bisphosphonates: sacrificing the jaw to save the skeleton? Bonekey Osteovision 2006;3:12-8

35. Lurie AG, Matteson SR. 99 M/Tc-diphosphonate bone imaging and uptake in healing rat extraction sockets. J Nucl Med 1976; 17:688-92.

36. McDougall IR. Skeletal scintigraphy. West J Med 1979;130:50314.

37. Hellstein JW, Marek CL. Bisphosphonate osteochemonecrosis (bis-phossy jaw): is this phossy jaw of the 21st century? J Oral Maxillofac Surg 2005;63:682-9.

38. Hansen T, Kunkel M, Springer E, Walter C, Weber A, Siegel E, et al. Actinomycosis of the jaws-histopathological study of 45 patients shows significant involvement in bisphosphonate-associated osteonecrosis and infected osteoradionecrosis. Virchows Arch 2007;451:1009-17.

39. Khamaisi M, Regev E, Yarom N, Avni B, Leitersdorf E, Raz I, et al. Possible association between diabetes and bisphosphonate-related jaw osteonecrosis. J Clin Endocrinol Metab 2007;92:11725 .

40. Wessel JH, Dodson TB, Zavras AI. Zoledronate, smoking and obesity are strong risk factors for osteonecrosis of the jaw: a case-control study. J Oral Maxillofac Surg 2008;66:625-31.

41. Bamias A, Kastritis E, Bamia C, Moulopoulos LA, Melakopoulos I, Bozas G, et al. Osteonecrosis of the jaw in cancer after treatment with bisphosphonates: incidence and risk factors. J Clin Oncol 2005;23:8580-7.

42. Hoff AO, Toth BB, Altundag K, Guarneri V, Adamus A, Nooka $\mathrm{AK}$, et al. Osteonecrosis of the jaw in patients receiving intravenous bisphosphonate therapy. ASCO Annual Meeting Proceedings (postmeeting edition). J Clin Oncol 2006;24(18 Suppl):8528. 
43. Durie BG, Katz M, Crowley J. Osteonecrosis of the jaws and bisphosphonates. N Engl J Med 2005;353:99-102.

44. Badros A, Weikel D, Salama A, Goloubeva O, Schneider A, Rapoport A, et al. Osteonecrosis of the jaw in multiple myeloma patients: clinical features and risk factors. J Clin Oncol 2006; 24:945-52.

45. Sarasquete ME, García-Sanz R, Marín L, Alcoceba M, Chillón MC, Balanzategui A, et al. Bisphosphonate-related osteonecrosis of the jaw is associated with polymorphisms of the cytochrome P450 CYP2C8 in multiple myeloma: a genome-wide single nucleotide polymorphism analysis. Blood 2008;112:2709-12.

46. Krane SM. Death and taxes: glucocorticoids and Bone Cell Apoptosis. BoneKEy-Osteovision 2001;1:2001019.

47. Ruggiero SL, Drew SJ. Osteonecrosis of the jaws and bisphosphonate therapy. J Dent Res 2007;86:1013-21.

48. Ruggiero SL, Fantasia J, Carlson E. Bisphosphonate-related osteonecrosis of the jaw: background and guidelines for diagnosis, staging and management. Oral Surg Oral Med Oral Pathol Oral Radiol Endod 2006;102:433-41.

49. Arce K, Assael LA, Weissman JL, Markiewicz MR. Imagind findings in bisphosphonate-related osteonecrosis of jaws. J Oral Maxillofac Surg 2009;67(5 Suppl):75-84.

50. Raje N, Woo SB, Hande K, Yap JT, Richardson PG, Vallet S, et al. Clinical, radiographic, and biochemical characterization of multiple myeloma patients with osteonecrosis of the jaw. Clin Cancer Res 2008;14:2387-95.

51. Marx RE, Cillo JE Jr, Ulloa JJ. Oral bisphosphonate-induced osteonecrosis: risk factors, prediction of risk using serum CTX testing, prevention, and treatment. J Oral Maxillofac Surg 2007;65: 2397-410.

52. Kunchur R, Need A, Hughes T. Clinical investigation of C-terminal cross-linking telopeptide test in prevention and management of bisphosphonate-associated osteonecrosis of the jaws. J Oral Maxillofac Surg 2010 68(2):487-8.

53. Kos M, Kuebler JF, Luczak K, Engelke W. Bisphosphonate-related osteonecrosis of the jaws: a review of 34 cases and evaluation of risk. J Craniomaxillofac Surg 2010;38:255-9.

54. Madrid C, Sanz M. What impact do systemically administrated bisphosphonates have on oral implant therapy? A systematic review. Clin Oral Implants Res 2009;20 Suppl 4:87-95. 